PROCEEDINGS OF THE

AMERICAN MATHEMATICAL SOCIETY

Volume 131, Number 4, Pages 1015-1020

S 0002-9939(02)06607-8

Article electronically published on June 12, 2002

\title{
REDUCTION NUMBERS AND INITIAL IDEALS
}

\author{
ALDO CONCA
}

(Communicated by Wolmer V. Vasconcelos)

\begin{abstract}
The reduction number $r(A)$ of a standard graded algebra $A$ is the least integer $k$ such that there exists a minimal reduction $J$ of the homogeneous maximal ideal $\mathbf{m}$ of $A$ such that $J \mathbf{m}^{k}=\mathbf{m}^{k+1}$. Vasconcelos conjectured that $r(R / I) \leq r(R / \operatorname{in}(I))$ where in $(I)$ is the initial ideal of an ideal $I$ in a polynomial ring $R$ with respect to a term order. The goal of this note is to prove the conjecture.
\end{abstract}

\section{REDUCTION NUMBERS AND INITIAL IDEALS}

Let $K$ be an infinite field and let $A=\bigoplus_{i \in \mathbf{N}} A_{i}$ be a homogeneous $K$-algebra, that is, an algebra of the form $R / I$ where $R=K\left[x_{1}, \ldots, x_{n}\right]$ is a polynomial ring and $I$ is a homogeneous ideal. The reduction number $r(A)$ of $A$ is the least integer $k$ such that there exists a minimal reduction $J$ of the homogeneous maximal ideal $\mathbf{m}$ of $A$ such that $J \mathbf{m}^{k}=\mathbf{m}^{k+1}$. It is not difficult to see that $r(A)$ is the largest integer $k$ such that the Hilbert function of $A / J$ at $k$ does not vanish; here $J$ is the ideal of $A$ generated by $d=\operatorname{dim} A$ generic linear forms.

Vasconcelos conjectured [10, Conjecture 7.2] that

$$
r(R / I) \leq r\left(R / \operatorname{in}_{\tau}(I)\right)
$$

where $\operatorname{in}_{\tau}(I)$ is the initial ideal of $I$ with respect to a term order $\tau$. The conjecture has been proved by Bresinsky and Hoa [2] for the generic initial ideal $\operatorname{Gin}_{\tau}(I)$, or, more generally, when $\operatorname{in}_{\tau}(I)$ is Borel-fixed. Trung 8] showed that $r(R / I)=$ $r\left(R / \operatorname{Gin}_{\mathrm{RL}}(I)\right)$ where $\operatorname{Gin}_{\mathrm{RL}}(I)$ is the generic initial ideal of $I$ with respect to the degree reverse lexicographic order RL (revlex for short).

The goal of this note is to prove the conjecture in general. After this paper was written we were informed that Trung [9] has independently solved the conjecture in general by a completely different method. What we prove is the following generalization of Vasconcelos' conjecture:

Theorem 1.1. Let $p$ be an integer, $0 \leq p \leq n$, and let $\operatorname{in}_{\tau}(I)$ be the initial ideal of I with respect to a term order $\tau$. Let $J$ be an ideal generated by $p$ generic linear forms. Then the Hilbert function of $R / I+J$ is $\leq$ that of $R / \operatorname{in}_{\tau}(I)+J$, that is,

$$
\operatorname{dim}_{K}[R / I+J]_{j} \leq \operatorname{dim}_{K}\left[R / \operatorname{in}_{\tau}(I)+J\right]_{j}
$$

for all $j \in \mathbf{N}$.

Received by the editors September 24, 2001 and, in revised form, October 29, 2001.

2000 Mathematics Subject Classification. Primary 13P10, 13A30; Secondary 13F20.

Key words and phrases. Gröbner bases, initial ideal, reduction number, Lex-segment ideal.

(C)2002 American Mathematical Society 
Taking $p=\operatorname{dim} R / I$ and $j=r\left(R / \operatorname{in}_{\tau}(I)\right)+1$ one obtains $r(R / I)<j$ which implies Vasconcelos' conjecture. To prove Theorem 1.1 we need some preparation.

Lemma 1.2. Let $p$ be an integer, $0 \leq p \leq n$, and let $J$ be an ideal generated by $p$ generic linear forms. Then the Hilbert function of $R / I+J$ is equal to the Hilbert function of $R / \operatorname{Gin}_{\mathrm{RL}}(I)+H$ where $\operatorname{Gin}_{\mathrm{RL}}(I)$ is the revlex Gin of $I$ and $H=\left(x_{n-p+1}, x_{n-p+2}, \ldots, x_{n}\right)$.

Proof. Set $J=\left(y_{1}, \ldots, y_{p}\right)$. We take a matrix $g \in \mathrm{GL}_{n}(K)$ such that the induced $K$-algebra graded homomorphism $g: R \rightarrow R$ maps $y_{i}$ to $x_{n-p+i}$ for $i=1, \ldots, p$. It follows that the Hilbert function of $R / I+J$ equals that of $R / g(I)+H$. Taking initial ideals does not change the Hilbert function and by the known properties of the revlex order one has $\operatorname{in}_{\mathrm{RL}}(g(I)+H)=\mathrm{in}_{\mathrm{RL}}(g(I))+H$. But since the $y_{i}$ are generic, $g$ is generic as well. Then $\operatorname{in}_{\mathrm{RL}}(g(I))=\operatorname{Gin}_{\mathrm{RL}}(I)$ and we are done.

We would now like to compare $\operatorname{Gin}_{\mathrm{RL}}(I)$ with $\operatorname{Gin}_{\mathrm{RL}}\left(\operatorname{in}_{\tau}(I)\right)$. To this end, let us introduce a piece of notation.

Let $V \subset R_{i}$ be a subspace of forms of degree $i$ and dimension $d$. Then $\bigwedge^{d} V$ is a subspace of dimension 1 of $\bigwedge^{d} R_{i}$. We identify in the following $\bigwedge^{d} V$ with any non-zero element contained in it. Fix a term order $<_{\sigma}$ in $R$. An exterior monomial is an element of the form $m_{1} \wedge \cdots \wedge m_{d}$ where the $m_{j}$ are distinct monomials of $R_{i}$. An exterior monomial $m_{1} \wedge \cdots \wedge m_{d}$ is $\sigma$-standard if $m_{1}>_{\sigma} \cdots>_{\sigma} m_{d}$. Note that $\bigwedge^{d} R_{i}$ has a basis consisting of the $\sigma$-standard exterior monomials. We order the $\sigma$-standard exterior monomials lexicographically:

$$
m_{1} \wedge \cdots \wedge m_{d}>_{\sigma} n_{1} \wedge \cdots \wedge n_{d}
$$

if $m_{i}>_{\sigma} n_{i}$ for the smallest index $i$ such that $m_{i} \neq n_{i}$. Then one defines the initial (exterior) monomial with respect to $\sigma$ of any element $f$ in the exterior space $\bigwedge^{d} R_{i}$ and the initial subspace of any subspace of $\bigwedge^{d} R_{i}$. By construction one has that $\operatorname{in}_{\sigma}(V)=\left\langle m_{1}, \ldots, m_{d}\right\rangle$ and $m_{1}>_{\sigma} \cdots>_{\sigma} m_{d}$ if and only if $\operatorname{in}_{\sigma}\left(\bigwedge^{d} V\right)=$ $m_{1} \wedge \cdots \wedge m_{d}$. For an element $F \in \wedge^{d} R_{i}$ we define its $\sigma$-support $\operatorname{Support}_{\sigma}(F)$ to be the set of the $\sigma$-standard exterior monomials which appear with a non-zero coefficient in $F$. Note that any exterior monomial $n$ is equal (up to sign) to a $\sigma$-standard exterior monomial. Note also that given an element $F \in \wedge^{d} R_{i}$ and two term orders $\sigma$ and $\tau$, the $\tau$-support of $F$ is obtained by taking the $\tau$-standard form of the elements in $\operatorname{Support}_{\sigma}(F)$. One has:

Lemma 1.3. Let $\sigma$ be a term order. Let $m=m_{1} \wedge \cdots \wedge m_{d}$ be a $\sigma$-standard exterior monomial, and let $q=q_{1} \wedge \cdots \wedge q_{d}$ be an exterior monomial with $q_{i} \leq_{\sigma} m_{i}$ for $i=1, \ldots, d$. Let $n=n_{1} \wedge \cdots \wedge n_{d}$ be the $\sigma$-standard exterior monomial corresponding to $q$. Then $n_{i} \leq_{\sigma} m_{i}$ for $i=1, \ldots, d$.

Proof. Since $n$ is obtained from $q$ by a sequence of transposition exchanging $q_{j}$ with $q_{j+1}$ whenever $q_{j}<_{\sigma} q_{j+1}$, it suffices to check that the property $q_{i} \leq_{\sigma} m_{i}$ for all $i$ is preserved by any such transposition. This is clear since $m_{j}>_{\sigma} m_{j+1} \geq_{\sigma} q_{j+1}$ and $m_{j+1} \geq_{\sigma} q_{j+1}>_{\sigma} q_{j}$.

Lemma 1.4. Let $\sigma$ be a term order. Let $V$ be a subspace of $R_{i}$ of dimension $d$, and let $\operatorname{in}_{\sigma}\left(\bigwedge^{d} V\right)=m_{1} \wedge \cdots \wedge m_{d}$. For every $n_{1} \wedge \cdots \wedge n_{d} \in \operatorname{Support}_{\sigma}\left(\bigwedge^{d} V\right)$ one has $m_{i} \geq_{\sigma} n_{i}$ for $i=1, \ldots, d$. 
Proof. Let $f_{1}, \ldots, f_{d}$ be elements in $V$ such that $\operatorname{in}\left(f_{i}\right)=m_{i}$. Then $\bigwedge^{d} V=$ $f_{1} \wedge \cdots \wedge f_{d}$. For $i=1, \ldots, d$ let $q_{i}$ be a monomial in $f_{i}$. It suffices to show that the $\sigma$-standard exterior monomial corresponding to $q_{1} \wedge \cdots \wedge q_{d}$ satisfies the desired property. This follows from Lemma 1.3 since $q_{i} \leq_{\sigma} m_{i}$.

The crucial fact is the following:

Lemma 1.5. Let $V$ be a d-dimensional subspace of $R_{i}$. Let $\sigma$ and $\tau$ be term orders. Set $W=\operatorname{in}_{\tau}(V)$. Let $g \in \mathrm{GL}_{n}(K)$ be a generic matrix acting as a $K$-algebra graded homomorphism on $R$. Then

$$
\operatorname{Support}_{\sigma}\left(g\left(\bigwedge^{d} W\right)\right) \subseteq \operatorname{Support}_{\sigma}\left(g\left(\bigwedge^{d} V\right)\right) .
$$

Proof. Let $W=\left\langle m_{1}, \ldots, m_{d}\right\rangle$ and let $f_{1}, \ldots, f_{d}$ in $V$ so that $\operatorname{in}_{\tau}\left(f_{i}\right)=m_{i}$. Set $F=f_{1} \wedge \cdots \wedge f_{d}$ and $M=m_{1} \wedge \cdots \wedge m_{d}$. We have to show that $\operatorname{Support}_{\sigma}(g(M)) \subseteq$ $\operatorname{Support}_{\sigma}(g(F))$. The matrix $g$ acts on $R$ by, say, $g\left(x_{i}\right)=\sum_{j} g_{i j} x_{j}$. We give to $g_{i j}$ a multidegree: $\operatorname{deg}\left(g_{i j}\right)=e_{i} \in \mathbf{Z}^{n}$. In the following $\log (m)$ denotes the exponent of a monomial $m$. For any monomial $m$ of $R_{i}$ we have that $g(m)$ is a sum of monomials of $R_{i}$ whose coefficients are polynomials of degree $\log (m)$ in the $g_{i j}$. Similarly, if $n=n_{1} \wedge \cdots \wedge n_{d}$ is an exterior monomial, then $g(n)$ is a sum of $\sigma$-standard exterior monomials whose coefficients are polynomials in the $g_{i j}$ of degree $\log \left(n_{1} \cdots n_{d}\right)$. Now assume $n=n_{1} \wedge \cdots \wedge n_{d}$ is a $\sigma$-standard exterior monomial in the $\sigma$-support of $g(M)$. If $n$ arises in the expansion of $g(Q)$ where $Q=q_{1} \wedge \cdots \wedge q_{d}$ for monomials $q_{i}$ in the support of $f_{i}$, then the coefficient of $n$ in $g(Q)$ is a polynomial of degree $\log \left(q_{1} \ldots q_{d}\right)$ in the $g_{i j}$. If at least one of the $q_{i}$, say $q_{j}$, is $<_{\tau} m_{i}$, then $q_{1} \ldots q_{d}<_{\tau} m_{1} \ldots m_{d}$. In particular $q_{1} \ldots q_{d} \neq m_{1} \ldots m_{d}$. It follows that the coefficients of $n$ in $g(M)$ and in $g(Q)$ are polynomials in the $g_{i j}$ of different degree. Therefore the coefficient of $n$ in $g(F)$ is a multi-homogeneous polynomial in the $g_{i j}$ and one of its homogeneous components is exactly the coefficient of $n$ in $g(M)$. This suffices to show that, for a generic $g$, the element $n$ is in the $\sigma$-support of $g(F)$.

Corollary 1.6. Let $V$ be a d-dimensional subspace of $R_{i}$. Let $\tau$ and $\sigma$ be term orders. Let $\operatorname{Gin}_{\sigma}(V)=\left\langle m_{1}, \ldots, m_{d}\right\rangle$ and $\operatorname{Gin}_{\sigma}\left(\operatorname{in}_{\tau}(V)\right)=\left\langle n_{1}, \ldots, n_{d}\right\rangle$ with $\left.m_{i}\right\rangle_{\sigma}$ $m_{i+1}$ and $n_{i}>_{\sigma} n_{i+1}$ for all $i=1, \ldots, d-1$. Then $m_{i} \geq_{\sigma} n_{i}$ for all $i=1, \ldots, d$.

Proof. Set $W=\operatorname{in}_{\tau}(V), m=m_{1} \wedge \cdots \wedge m_{d}$ and $n=n_{1} \wedge \cdots \wedge n_{d}$. By construction $\operatorname{in}_{\sigma}\left(g\left(\wedge^{d} W\right)\right)=n$ for a generic matrix $g$. By virtue of Lemma 1.5 $n \in \operatorname{Support}_{\sigma}\left(g\left(\wedge^{d} V\right)\right)$ and by construction $\operatorname{in}_{\sigma}\left(g\left(\wedge^{d} V\right)\right)=m$. It follows from Lemma 1.4 that $n_{i} \leq_{\sigma} m_{i}$ for all $i=1, \ldots, d$.

We are ready to prove Theorem 1.1

Proof of Theorem 1.1. Set $H=\left(x_{n-p+1}, x_{n-p+2}, \ldots, x_{n}\right)$. By virtue of Lemma 1.2 it is enough to show that the Hilbert function of $R / \operatorname{Gin}_{\mathrm{RL}}(I)+H$ is less than or equal to that of $R / \operatorname{Gin}_{\mathrm{RL}}\left(\operatorname{in}_{\tau}(I)\right)+H$. Fix an integer $j$ and set

$$
a=\operatorname{dim}\left[R / \operatorname{Gin}_{\mathrm{RL}}(I)+H\right]_{j} \quad \text { and } \quad b=\operatorname{dim}\left[R / \operatorname{Gin}_{\mathrm{RL}}\left(\operatorname{in}_{\tau}(I)\right)+H\right]_{j} .
$$

We must show that $a \leq b$. Let $V$ be the component of degree $j$ of $I$. Set $d=\operatorname{dim} V$, $\operatorname{Gin}_{\mathrm{RL}}(V)=\left\langle m_{1}, \ldots, m_{d}\right\rangle, \operatorname{Gin}_{\mathrm{RL}}\left(\operatorname{in}_{\tau}(V)\right)=\left\langle n_{1}, \ldots, n_{d}\right\rangle$ and assume that $\left.m_{i}\right\rangle_{\mathrm{RL}}$ 
$m_{i+1}$ and $n_{i}>_{\mathrm{RL}} n_{i+1}$. For a monomial $m$ we set $\max (m)=\max \left\{i: x_{i}\right.$ divides $\left.m\right\}$. By construction we have

$$
b-a=\left|\left\{k: \max \left(m_{k}\right) \leq n-p\right\}\right|-\left|\left\{k: \max \left(n_{k}\right) \leq n-p\right\}\right| .
$$

By Corollary 1.6 we know that $m_{i} \geq_{\mathrm{RL}} n_{i}$ for all $i$. This implies that $\max \left(m_{i}\right) \leq$ $\max \left(n_{i}\right)$ for all $i$. Hence $\left\{k: \max \left(m_{k}\right) \leq n-p\right\} \supseteq\left\{k: \max \left(n_{k}\right) \leq n-p\right\}$ and we are done.

Remark 1.7. One easily checks that the proof of Theorem 1.1 also works if one takes the initial ideal with respect to a positive weight function $\omega$. In particular Vasconcelos' conjecture holds in this situation, too.

Remark 1.8. With the notation of Theorem 1.1 one could ask whether there is a relation between the graded Betti numbers of $R / I+J$ and those of $R / \operatorname{in}_{\tau}(I)+J$. The known properties of the initial ideal imply that the former are smaller than the latter for instance when $p \leq \operatorname{depth} R / \operatorname{in}_{\tau}(I)$. But this relation does not hold in general. This is because, as we know, the Hilbert function of $R / I+J$ is less than or equal to that of $R / \operatorname{in}_{\tau}(I)+J$ and hence the number of generators in low degrees of $I+J$ tends to be larger than that of $\operatorname{in}_{\tau}(I)+J$. For instance, taking $I=\left(x^{2}+y z, x y, x z\right)$ and $\tau$ to be the lex order, then $\operatorname{in}_{\tau}(I)=\left(x^{2}, x y, x z, y z^{2}, y^{2} z\right)$ and for a general linear form $L$ the ideal $I+(L)$ has 3 minimal generators in degree 2 , while $\operatorname{in}_{\tau}(I)+(L)$ has only 2 minimal generators in degree 2 .

Remark 1.9. Recall that the analytic spread $\ell(I)$ of an ideal $I$ is the Krull dimension of the fiber ring $\bigoplus_{i=0}^{\infty} I^{i} / \mathbf{m} I^{i}$. One can ask whether there is a relation between the analytic spread $I$ and that of $\operatorname{in}_{\tau}(I)$. There are examples where $\ell(I)>\ell\left(\operatorname{in}_{\tau}(I)\right)$ and others where $\ell(I)<\ell\left(\operatorname{in}_{\tau}(I)\right)$. As for the former, take for instance the ideal $I$ of the 2 -minors of a generic $3 \times 3$ symmetric matrix and $\tau$ a diagonal term order (i.e. the initial term of a minor is the product of the elements of the main diagonal). One has $\ell(I)=6$ and $\ell\left(\operatorname{in}_{\tau}(I)\right)=5$. On the other hand, if $I$ is the ideal generated by 2 generic quadrics in 3 variables and $\tau$ is the lex order, then $\ell(I)=2$ and $\ell\left(\operatorname{in}_{\tau}(I)\right)=3$.

\section{ReduCtion Numbers AND LEX-SEGMENT IDEALS}

A monomial ideal $L$ of $R$ is said to be a Lex-segment if whenever $m$ is a monomial in $L$ and $n$ is a monomial with $\operatorname{deg}(n)=\operatorname{deg}(m)$ and $n>m$ with respect to the lexicographic order, then one has that $n \in L$. Given a homogeneous ideal $I$ there is a unique Lex-segment ideal $I^{\text {Lex }}$ such that the Hilbert function of $I^{\text {Lex }}$ is equal to that of $I$. It is well-know that $I^{\text {Lex }}$ is "extremal" with respect to many invariants in the class of the ideals with a given Hilbert function (e.g. absolute Betti numbers Bigatti [1], Hulett [4 and Pardue 6], relative Betti numbers Iyengar and Pardue [5], local cohomology Sbarra [7], etc.). Therefore it is natural to ask whether the same also holds for the reduction number, i.e. whether $r(R / I) \leq r\left(R / I^{\mathrm{Lex}}\right)$ holds in general. We have:

Proposition 2.1. If $K$ has characteristic 0 , then

$$
r(R / I) \leq r\left(R / I^{\mathrm{Lex}}\right)
$$

holds for every homogeneous ideal of $I$ of $K\left[x_{1}, \ldots, x_{n}\right]$. 
Proof. Let $I$ be a homogeneous ideal of $R=K\left[x_{1}, \ldots, x_{n}\right]$ and set $d=\operatorname{dim} R / I$ and $J=\operatorname{Gin}_{\mathrm{RL}}(I)$. It is know that $J$ is Borel-fixed, that is, fixed under the action of the group of the upper-triangular matrix. In characteristic 0 this is equivalent to saying that $J$ is strongly stable, that is, if $x_{i} m$ is a monomial in $J$ and $j<i$, then $x_{j} m$ is in $J$ as well. From this and from Lemma 1.2 it follows immediately that if char $K=0$, then $r(R / I)$ is equal to the least integer $k$ such that $x_{n-d}^{k+1}$ is in $J$ (this has also been observed in [8]). Then the desired inequality is a consequence of the following fact:

Claim. Let $V$ and $L$ be sets of monomials of degree $k$ with the same cardinality such that $V$ is strongly stable and $L$ is a Lex-segment. If $x_{i}^{k} \in L$ for some $i$, then $x_{i}^{k} \in V$.

To prove the claim one observes that since $L$ contains $x_{i}^{k}$ and is a Lex-segment, then $L$ also contains the set, say $A$, of all the monomials of degree $k$ which are divisible by some $x_{j}$ with $j<i$. Therefore $|L| \geq|A|+1$. Since $|V|=|L|$ it follows that $V$ contains a monomial $m$ which is not in $A$. In other words, $V$ contains a monomial supported only on the variables $x_{i}, x_{i+1}, \ldots, x_{n}$. Since $V$ is stable, then $V$ also contains $x_{i}^{k}$.

We believe that the inequality of Proposition 2.1 is also true if the characteristic of the base field is finite. Pardue developed in [6] a characteristic-free strategy for proving that the Lex-segment ideal is extremal with respect to a certain invariant, say $\alpha(R / I)$. Roughly speaking, it says that if $\alpha$ does not decrease by taking initial ideals and also does not decrease by performing a certain deformation process, called polarization, then one has $\alpha(R / I) \leq \alpha\left(R / I^{\text {Lex }}\right)$ for all the ideals $I$. For the definition of polarization of a monomial ideal we refer the reader to the paper of Pardue [6]. Unfortunately one cannot use Pardue's argument to prove the above inequality. This is because the reduction number can decrease under polarizations. For example, let $R=K\left[x_{1}, \ldots, x_{4}\right]$ and

$$
I=\left(x_{4}^{2}, x_{1} x_{3}^{3}, x_{3}^{3} x_{4}, x_{2}^{3} x_{4}, x_{2} x_{3}^{3}, x_{2}^{3} x_{3}, x_{1}^{2} x_{3}^{2}, x_{1}^{4}, x_{1} x_{2}^{2} x_{4}, x_{2}^{4}\right)
$$

and $J$ its polarization; one can check that $r(R / I)=4$ and $r(R / J)=3$. In this case $r\left(R / I^{\text {Lex }}\right)=5$.

Let us also note that the above ideal can be used to construct an example of a standard graded algebra $A$ and a non-zero divisor $z$ of degree 1 such that $r(A)<r(A / z A)$. To this end it suffices to take $S=K\left[x_{1}, \ldots, x_{5}\right]$, and

$$
I_{1}=\left(x_{4} x_{5}, x_{1} x_{3}^{3}, x_{3}^{3} x_{4}, x_{2}^{3} x_{4}, x_{2} x_{3}^{3}, x_{2}^{3} x_{3}, x_{1}^{2} x_{3}^{2}, x_{1}^{4}, x_{1} x_{2}^{2} x_{4}, x_{2}^{4}\right) .
$$

In other words, $I_{1}$ is the polarization of the ideal $I$ above with respect to the variable $x_{4}$. Set $A=S / I_{1}$ and $z=x_{4}-x_{5}$. Then $z$ is a non-zero divisor of $A$ and $r(A)=3$ and $r(A / z A)=4$.

\section{ACKNOWLEDGEMENT}

We would like to thank Marilina Rossi for useful discussions concerning the material of this paper. The explicit examples that we have presented in the paper have been detected by using the computer algebra system CoCoA [3].

\section{REFERENCES}

1. A. Bigatti, Upper bounds for the Betti numbers of a given Hilbert function, Comm. Algebra 21 (1993), no. 7, 2317-2334. MR 94c:13014 
2. H. Bresinsky, L. Hoa, On the reduction number of some graded algebras, Proc. Amer. Math. Soc. 127 (1999), no. 5, 1257-1263. MR 99h:13027.

3. A. Capani, G. Niesi, L. Robbiano, CoCoA, a system for doing Computations in Commutative Algebra, Available ftp from cocoa.dima.unige.it.

4. H. Hulett, Maximum Betti numbers of homogeneous ideals with a given Hilbert function, Comm. Algebra 21 (1993), no. 7, 2335-2350. MR 94c:13015

5. S. Iyengar, K. Pardue, Maximal minimal resolutions, J. Reine Angew. Math. 512 (1999), 27-48. MR 2000d:13023

6. K. Pardue, Deformation classes of graded modules and maximal Betti numbers, Illinois J. Math. 40 (1996), no. 4, 564-585. MR 97g:13029

7. E. Sbarra, Upper bounds for local cohomology for rings with given Hilbert function, Comm. Algebra 29 (2001), 5383-5409.

8. N. Trung, Gröbner bases, local cohomology and reduction number, Proc. Amer. Math. Soc. 129 (2001), no. 1, 9-18. MR 2001c:13042

9. N. Trung, Constructive characterization of the reduction numbers, preprint 2001.

10. W. Vasconcelos, Cohomological degrees of graded modules, in Six lectures on commutative algebra (Bellaterra, 1996), 345-392, Progr. Math., 166, Birkhäuser, Basel, 1998. MR 99j:13012

Dipartimento di Matematica, Universitá di Genova, Via Dodecaneso 35, I-16146 GenOVA, ITALIA

E-mail address: conca@dima.unige.it 